\title{
Crescimento do fungo simbionte de formigas cortadeiras do gênero Acromyrmex em meios de cultura com diferentes extratos
}

\author{
Growth of symbiont fungi of cutter ants of the genus Acromyrmex in means of culture with different extracts
}

\author{
Regina da Silva Borba ${ }^{1 *}$ Alci Enimar Loeck ${ }^{1}$ Juliana de Magalhães Bandeira² ${ }^{2}$ Caroline Leivas Moraes ${ }^{2}$ \\ Elias Daniel Centenaro ${ }^{1}$
}

\section{RESUMO}

As formigas cortadeiras mostram preferência por algumas espécies vegetais, as quais são constantemente desfolhadas, enquanto outras não são atacadas, embora sejam abundantes e localizadas próximas ao ninho. As formigas dos gêneros Atta e Acromyrmex cortam plantas e transportam pedaços para os formigueiros. O material vegetal é usado para o cultivo do fungo Leucoagaricus gongylophorus, do qual se alimentam. Vários trabalhos utilizando extratos de plantas têm sido realizados para avaliar o efeito sobre o desenvolvimento do fungo simbionte. O objetivo deste trabalho foi avaliar a influência de meios de cultura acrescidos de diferentes extratos sobre o crescimento do fungo $\boldsymbol{L}$. gongylophorus. Fungos das espécies Acromyrmex ambiguus, Acromyrmex crassispinus, Acromyrmex heyeri e Acromyrmex lundi foram inoculados nos meios Ágar Nutritivo e BDA, ambos acrescidos de melaço e de extratos de azevém (Lolium multiflorum Lam.), tifa (Typha angustifolia L.) e formiga (Atta sexdens piriventris). O meio Pagnocca foi utilizado como padrão. As placas foram mantidas em câmaras climatizadas à temperatura de $25+1^{\circ} \mathrm{C}$ e escotofase de $24 \mathrm{~h}$, por um período de 49 dias. $O$ crescimento fúngico foi avaliado com base no diâmetro da colônia, sendo este avaliado em intervalos semanais, totalizando sete avaliações. Também foi avaliado o peso seco do fungo aos 53 dias. Os resultados mostraram que o fungo cultivado por diferentes espécies de formigas tem crescimento diferenciado em meios de cultura com diferentes extratos.

Palavras-chave: Leucoagaricus gongylophorus, Lolium multiflorum, simbiose, Typha angustifolia.

\section{ABSTRACT}

The cutter ants show preference for some vegetable species, which are constantly defoliated, while others are not attacked, although they are abundant and located close to the nest. The ants of the goods Atta and Acromyrmex cut plants and they transport pieces to the anthills. The vegetable material is used for the cultivation of the mushroom Leucoagaricus gongylophorus, mushroom from which they feed. Several works using extracts of plants have been accomplished to evaluate the effect on the development of the mushroom symbiont. The objective of this work was to evaluate the influence of added culture means of different extracts on the growth of the $\mathbf{L}$. gongylophorus mushroom. Where mushrooms of the species Acromyrmex ambiguus, Acromyrmex crassispinus, Acromyrmex heyeri and Acromyrmex lundi were inoculated in the means Nutritious Ágar and BDA, both added of molasses and extracts of Lolium multiflorum Lam., Typha angustifolia L. and ant (Atta sexdens piriventris). The Pagnocca medium was used as pattern. The plates were maintained in cameras acclimatized to the temperature of $25+1^{\circ} \mathrm{C}$ and 24 hours of darkness, for a period of 49 days. The fungal growth was evaluated with base in the diameter of the colony, which was accomplished with weekly intervals, totalizing seven evaluations. Also the dry weight was evaluated from the mushroom to the 53 days. The results showed that the mushroom cultivated by different species of ants has differentiated growth in culture means with different extracts.

Key words: Leucoagaricus gongylophorus, Lolium multiflorum, symbiosis, Typha angustifolia.

\section{INTRODUÇÃo}

As formigas cortadeiras, pertencentes à tribo Attini, situam-se entre as piores pragas da agricultura brasileira. Num ecossistema natural, as formigas mostram preferência por algumas espécies vegetais,

\footnotetext{
${ }^{1}$ Departamento de Fitossanidade, Universidade Federal de Pelotas (UFPel), Campus Universitário, Caixa Postal 354, 96010-900, Pelotas, RS, Brasil.*E-mail: rsborba@pop.com.br

${ }^{2}$ Instituto de Biologia, UFPel, Pelotas, RS, Brasil.
} 
as quais são constantemente desfolhadas, enquanto outras não são atacadas, embora sejam abundantes e localizadas próximas ao ninho.

Observa-se que as variações na coleta de material vegetal estão relacionadas com as diferentes espécies de formigas. Umas têm preferência por espécies vegetais monocotiledôneas, outras por dicotiledôneas e algumas preferem ambas. Provavelmente, a preferência por espécies vegetais está relacionada com a exigência nutricional do fungo (Leucoagaricus gongylophorus) que cultivam. Dessa forma, é provável que os fungos cultivados pelas diversas espécies de formigas apresentem variações subespecíficas ou até mesmo específicas (JURUENA \& CACHAPUZ, 1980).

As formigas cultivadoras de fungos são “verdadeiras agricultoras”, capazes de manipular o fungo sobre o substrato vegetal para otimizar o seu crescimento. Transplantam o fungo de jardins velhos para novos especialmente preparados para acolhê-lo, onde otimizam seu crescimento através da aplicação regular de suas fezes (MARTIN, 1987; CHERRETT et al., 1989), da limpeza dos esporos contaminantes e da extirpação dos micélios infestados (CURRIE \& STUART, 2001).

Vários trabalhos com extratos de plantas para verificar o efeito sobre o desenvolvimento do fungo simbionte têm sido realizados. Um estudo realizado com macerados de folhas de Citrus spp., Ligustrum spp., Acalypha spp., Eucalyptus spp., Alchornea triplinervia e Melia spp. adicionados ao meio de cultura mostrou que as plantas maceradas afetaram diretamente o desenvolvimento do fungo simbionte (CAMARGO et al., 2003). KHOURI et al. (2003), avaliando o efeito de extratos de gramíneas forrageiras no crescimento do fungo simbionte de Acromyrmex balzani, concluíram que o capim tifton (Cynodon dactylon) e o capim pangola (Digitaria decumbens) promoveram melhor crescimento do fungo.

O fungo simbionte das formigas cortadeiras possui um crescimento muito lento em laboratório, dificultando as pesquisas realizadas. Por esse motivo, este trabalho foi realizado com o objetivo de avaliar o desenvolvimento do fungo simbionte $\boldsymbol{L}$. gongylophorus em meios de cultura acrescidos de melaço e de extratos de azevém (Lolium multiflorum Lam.), de tifa (Typha angustifolia L.) e de formiga (Atta sexdens piriventris), para saber qual meio possibilitaria um maior crescimento do referido fungo.

\section{MATERIAL E MÉTODOS}

O fungo foi coletado de ninhos de campo de Acromyrmex ambiguus, Acromyrmex crassispinus, Acromyrmex heyeri e Acromyrmex lundi, no município de Capão do Leão, RS.

Foram utilizados os meios de cultura: Ágar nutritivo (peptona 5g, extrato de carne 3g, cloreto de sódio 1g, ágar 15g, água destilada 1000ml) e BDA (batata $140 \mathrm{~g}$, dextrose $10 \mathrm{~g}$, ágar $15 \mathrm{~g}$, água destilada $1000 \mathrm{ml}$ ), cada um acrescido de melaço e de extratos de azevém, tifa e formiga. Utilizou-se o meio de cultivo rotineiro no laboratório, Pagnocca (glucose 10g, cloreto de sódio $5 \mathrm{~g}$, bacto peptona $5 \mathrm{~g}$, extrato de malte $10 \mathrm{~g}$, ágar $15 \mathrm{~g}$, água destilada 1000ml) (PAGNOCCA et al., 1990), como padrão. A utilização de extrato de formigas se deu devido à importância que estas possuem no cultivo desse fungo em seus ninhos, já que, segundo DELLA LUCIA (1993), as operárias fertilizam o substrato com suas excretas ricas em substâncias necessárias ao crescimento e desenvolvimento rápido do fungo.

Cada um dos nove tratamentos foi repetido seis vezes.

O extrato foi obtido triturando-se $100 \mathrm{~g}$ de folhas (azevém e tifa) ou de formigas vivas para $400 \mathrm{ml}$ de água destilada. Em seguida o extrato foi filtrado, sendo $20 \mathrm{ml}$ de seu conteúdo acrescidos a $100 \mathrm{ml}$ de meio de cultura. Em relação ao melaço, foram acrescidos $20 \mathrm{ml}$ deste diretamente no meio de cultura. Subseqüentemente, o material foi esterilizado a $121^{\circ} \mathrm{C}$ por 15 minutos e vertido em placas de Petri (VICTOR et al., 2001).

As placas de Petri foram inoculadas com discos de meios contendo micélio do fungo, utilizandose, para isso, um vazador de $4 \mathrm{~mm}$ de diâmetro. As placas foram mantidas em câmaras climatizadas do tipo BOD à temperatura de $25 \pm 1^{\circ} \mathrm{C}$ e escotofase de $24 \mathrm{~h}$, por um período de 49 dias. $\mathrm{O}$ crescimento fúngico foi avaliado macroscopicamente com base no diâmetro da colônia, em centímetros, sendo este avaliado em intervalos semanais, totalizando sete avaliações (aos 7, 14, 21, 28, 35, 42 e 49 dias após inoculação). Também foi avaliado o peso seco do fungo, em miligramas, aos 53 dias (se o fungo permanecer por muito mais tempo incubado, o meio pode secar e o fungo acaba morrendo), quando o micélio foi separado do meio de cultura através do uso de água fervente $\left(100 \pm 10^{\circ} \mathrm{C}\right)$. Aproximadamente $500 \mathrm{~mL}$ 
de água foram utilizados por placa de Petri. Após isso, procedeu-se à secagem em estufa a $60^{\circ} \mathrm{C}$ por 72 horas e à pesagem em balança analítica de precisão (NASCIMENTO, 2003).

O delineamento experimental foi inteiramente casualizado em esquema fatorial ( $9 \times 4 \times 7$ ) com nove meios de cultura, 4 procedências de fungos e 7 tempos de avaliação de diâmetro e (9 x 4) para a interação referente ao peso seco do fungo. Os dados obtidos foram testados quanto à normalidade e à homogeneidade das variâncias e, quando necessário, foram transformados em $\sqrt{(\mathrm{x}+0,5)}$, o que foi realizado para a variável peso seco do fungo. Como o fator tempo de avaliação é quantitativo, foram realizadas análises de regressão. As análises estatísticas foram realizadas através do Programa SAS (SAS Institute, 2000) e as médias comparadas pelo teste de Tukey, em nível de $5 \%$ de probabilidade de erro.

\section{RESULTADOS E DISCUSSÃO}

Nos tratamentos Ágar nutritivo + melaço e BDA + melaço, não houve crescimento do fungo e os dois tratamentos foram alijados da análise estatística.

Em relação ao fungo de $A$. ambiguus (Tabela 1 ), o maior diâmetro micelial foi verificado no tratamento Ágar nutritivo + tifa, sendo significativamente superior aos demais tratamentos na maioria dos períodos avaliados, assim como o fungo de $\boldsymbol{A}$. crassispinus (Tabela 2) que, com este mesmo tratamento, apresentou diâmetro de crescimento superior aos demais até os 42 dias. Porém, na última avaliação (49 dias), não houve diferença significativa entre os tratamentos. Os fungos cultivados pelas espécies $\boldsymbol{A}$. heyeri e $\boldsymbol{A}$. lundi apresentaram maior crescimento micelial também no tratamento Ágar nutritivo + tifa (Tabelas 3 e 4).

No entanto, com relação ao peso seco, o tratamento padrão (Pagnocca) se mostrou superior aos demais para o fungo cultivado pela maioria das espécies de formigas testadas, com exceção do fungo de $\boldsymbol{A}$. heyeri, sobre o qual não houve diferença estatística entre os tratamentos (Tabela 5). Nesse sentido, esses resultados corresponderam ao trabalho de LOECK et al. (2004), os quais avaliando quatro diferentes meios de cultura (Pagnocca, M \& S, V 8 Juice ágar e Celuloseasparagine) sobre o crescimento do fungo de Atta sexdens piriventris e $\boldsymbol{A}$. heyeri, observaram que, para ambas as espécies, o meio Pagnocca apresentou o maior crescimento fúngico.

Em relação à diferença de peso seco de micélio entre as espécies de formigas, esta foi observada apenas nos meios BDA + T e Pagnocca, sendo que no primeiro, os fungos de $\boldsymbol{A}$. ambiguus e $\boldsymbol{A}$. heyeri se mostraram superiores em relação aos demais. Porém, no segundo meio referido, justamente o fungo da espécie $\boldsymbol{A}$. heyeri mostrou peso inferior ao das demais espécies de formigas (Tabela 5). O que ressalta, mais uma vez, o modo de crescimento diferenciado deste fungo em relação à espécie de formiga que o cultiva.

Considerando os resultados obtidos neste trabalho, a constatação de um crescimento em diâmetro superior aos demais não implica necessariamente em um peso seco superior. De acordo com KOCK (1975), diferenças de crescimento podem ocorrer em duas ou três dimensões. Além disso, a constituição do meio de cultura utilizado pode influenciar no modo de crescimento do fungo.

Tabela 1 - Diâmetro do crescimento micelial $(\mathrm{cm})$, em 7 períodos, do fungo Leucoagaricus gongylophorus (Acromyrmex ambiguus) cultivado em diferentes meios de culturas. Laboratório de Myrmecologia, FAEM/UFPel. Pelotas, RS, 2004.

\begin{tabular}{|c|c|c|c|c|c|c|c|}
\hline Tratamentos & 7 dias & 14 dias & 21 dias & 28 dias & 35 dias & 42 dias & 49 dias \\
\hline $\mathrm{AN}+\mathrm{A}^{*}$ & $0,72 \mathrm{c}$ & $1,23 \mathrm{~b}$ & 1,77 bc & $2,25 \mathrm{c}$ & $2,72 \mathrm{~b}$ & 3,05 bc & $3,35 \mathrm{~b}$ \\
\hline $\mathrm{AN}+\mathrm{F}$ & $0,58 \mathrm{e}$ & $1,14 \mathrm{c}$ & $1,34 \mathrm{~d}$ & $1,96 \mathrm{~d}$ & $2,64 \mathrm{~b}$ & 2,94 bc & $3,38 \mathrm{~b}$ \\
\hline $\mathrm{AN}+\mathrm{T}$ & 0,87 a & $1,25 b$ & $2,28 \mathrm{a}$ & 2,93 a & $3,45 \mathrm{a}$ & 3,93 a & $4,28 \mathrm{a}$ \\
\hline $\mathrm{BDA}+\mathrm{A}$ & $0,75 \mathrm{~b}$ & $1,13 \mathrm{c}$ & $1,37 \mathrm{~d}$ & $1,75 \mathrm{e}$ & $1,98 \mathrm{c}$ & $2,67 \mathrm{c}$ & $3,17 \mathrm{~b}$ \\
\hline $\mathrm{BDA}+\mathrm{F}$ & $0,62 \mathrm{~d}$ & $0,87 \mathrm{~d}$ & $1,67 \mathrm{c}$ & $1,73 \mathrm{e}$ & $2,13 \mathrm{c}$ & $2,70 \mathrm{c}$ & $3,32 \mathrm{~b}$ \\
\hline $\mathrm{BDA}+\mathrm{T}$ & $0,72 \mathrm{c}$ & $1,27 \mathrm{~b}$ & $1,90 \mathrm{~b}$ & $2,50 \mathrm{~b}$ & $2,93 \mathrm{c}$ & $3,27 \mathrm{~b}$ & $3,52 \mathrm{~b}$ \\
\hline Pagnocca (controle) & $0,75 \mathrm{~b}$ & 1,35 a & 1,85 bc & $2,25 \mathrm{c}$ & $2,75 \mathrm{~b}$ & $3,27 \mathrm{~b}$ & 3,65ab \\
\hline CV (\%) & 9,61 & 13,27 & 8,30 & 7,78 & 9,70 & 12,03 & 12,17 \\
\hline
\end{tabular}

Médias seguidas pela mesma letra nas colunas não diferem entre si pelo teste de Tukey a 5\% de probabilidade de erro. * AN + A = Ágar nutritivo + Azevém; AN + F = Ágar nutritivo + Formiga; AN + T = Ágar nutritivo + Tifa; BDA + A = BDA + Azevém; BDA + F = BDA + Formiga; BDA + T = BDA + Tifa. 
Tabela 2 - Diâmetro do crescimento micelial (cm), em 7 períodos, do fungo Leucoagaricus gongylophorus (Acromyrmex crassispinus), cultivado em diferentes meios de culturas. Laboratório de Myrmecologia, FAEM/UFPel. Pelotas, RS, 2004.

\begin{tabular}{|c|c|c|c|c|c|c|c|}
\hline Tratamentos & 7 dias & 14 dias & 21 dias & 28 dias & 35 dias & 42 dias & 49 dias \\
\hline $\mathrm{AN}+\mathrm{A}^{*}$ & $0,60 \mathrm{c}$ & $0,97 \mathrm{~cd}$ & 1,52 bc & $1,97 \mathrm{ab}$ & $2,20 \mathrm{bc}$ & $2,92 \mathrm{ab}$ & $3,32 \mathrm{a}$ \\
\hline$A N+F$ & $0,58 \mathrm{~d}$ & $1,07 \mathrm{c}$ & $1,43 \mathrm{c}$ & $1,92 \mathrm{ab}$ & 2,18 bc & $2,58 \mathrm{ab}$ & 2,88 a \\
\hline $\mathrm{AN}+\mathrm{T}$ & 0,78 a & 1,30 a & 1,92 a & $2,24 \mathrm{a}$ & $2,92 \mathrm{a}$ & 3,40 a & 3,96 a \\
\hline $\mathrm{BDA}+\mathrm{A}$ & $0,58 \mathrm{e}$ & $0,90 \mathrm{~d}$ & $1,20 \mathrm{~d}$ & $1,30 \mathrm{c}$ & $1,92 \mathrm{c}$ & $2,30 \mathrm{~b}$ & 2,88 a \\
\hline $\mathrm{BDA}+\mathrm{F}$ & $0,57 \mathrm{f}$ & $0,97 \mathrm{~cd}$ & $1,37 \mathrm{~cd}$ & $1,73 \mathrm{bc}$ & 2,43abc & $2,90 \mathrm{ab}$ & $3,07 \mathrm{a}$ \\
\hline $\mathrm{BDA}+\mathrm{T}$ & $0,54 \mathrm{~g}$ & $1,20 \mathrm{ab}$ & 1,55 bc & $1,95 \mathrm{ab}$ & 2,25 bc & 2,63ab & $2,98 \mathrm{a}$ \\
\hline Pagnocca (controle) & $0,67 \mathrm{~b}$ & $1,18 \mathrm{~b}$ & $1,65 \mathrm{~b}$ & $2,10 a b$ & $2,55 \mathrm{ab}$ & $3,12 \mathrm{ab}$ & 3,50 a \\
\hline CV (\%) & 11,36 & 11,08 & 11,35 & 15,80 & 19,68 & 21,57 & 22,62 \\
\hline
\end{tabular}

Médias seguidas pela mesma letra nas colunas não diferem entre si pelo teste de Tukey a 5\% de probabilidade de erro. * AN + A $=$ Ágar nutritivo + Azevém; AN + F = Ágar nutritivo + Formiga; AN + T = Ágar nutritivo + Tifa; BDA + A = BDA + Azevém; BDA + F = BDA + Formiga; BDA + T = BDA + Tifa.

Tabela 3 - Diâmetro do crescimento micelial (cm), em 7 períodos, do fungo Leucoagaricus gongylophorus (Acromyrmex heyeri), cultivado em diferentes meios de culturas. Laboratório de Myrmecologia, FAEM/UFPel. Pelotas, RS, 2004.

\begin{tabular}{|c|c|c|c|c|c|c|c|}
\hline Tratamentos & 7 dias & 14 dias & 21 dias & 28 dias & 35 dias & 42 dias & 49 dias \\
\hline $\mathrm{AN}+\mathrm{A}^{*}$ & $0,77 \mathrm{a}$ & $1,33 \mathrm{~b}$ & $1,75 \mathrm{~b}$ & 2,27 bc & $2,47 \mathrm{c}$ & 2,95 bc & 3,08 bc \\
\hline $\mathrm{AN}+\mathrm{F}$ & $0,62 \mathrm{f}$ & $1,06 \mathrm{c}$ & $1,50 \mathrm{~b}$ & $2,14 \mathrm{c}$ & $2,68 \mathrm{bc}$ & 3,14 abc & 3,24 bc \\
\hline $\mathrm{AN}+\mathrm{T}$ & $0,75 \mathrm{~b}$ & $1,52 \mathrm{a}$ & 2,15 a & $2,67 \mathrm{ab}$ & $3,42 \mathrm{a}$ & 3,95 a & 4,27 a \\
\hline $\mathrm{BDA}+\mathrm{A}$ & $0,72 \mathrm{c}$ & $1,30 \mathrm{~b}$ & $1,74 \mathrm{~b}$ & $2,06 \mathrm{c}$ & $2,26 \mathrm{c}$ & $2,58 \mathrm{c}$ & $2,94 \mathrm{c}$ \\
\hline $\mathrm{BDA}+\mathrm{F}$ & $0,68 \mathrm{~d}$ & $1,30 \mathrm{~b}$ & $1,70 \mathrm{~b}$ & 2,28 bc & 2,60 bc & 3,10 abc & 3,56 abc \\
\hline $\mathrm{BDA}+\mathrm{T}$ & 0,77 a & $1,43 a b$ & 2,12 a & 2,78 a & $3,22 \mathrm{ab}$ & $3,57 a b$ & $3,87 \mathrm{ab}$ \\
\hline Pagnocca (controle) & 0,63 e & 0,98 c & $1,13 \mathrm{c}$ & $1,30 \mathrm{~d}$ & $1,52 \mathrm{~d}$ & $1,77 \mathrm{~d}$ & $1,93 \mathrm{~d}$ \\
\hline CV (\%) & 14,03 & 11,65 & 14,63 & 13,16 & 15,58 & 15,26 & 14,35 \\
\hline
\end{tabular}

Médias seguidas pela mesma letra nas colunas não diferem entre si pelo teste de Tukey a 5\% de probabilidade de erro. * AN + A = Ágar nutritivo + Azevém; AN + F = Ágar nutritivo + Formiga; AN + T = Ágar nutritivo + Tifa; BDA + A = BDA + Azevém; BDA + F = BDA + Formiga; BDA + T = BDA + Tifa.

Tabela 4 - Diâmetro do crescimento micelial (cm), em 7 períodos, do fungo Leucoagaricus gongylophorus (Acromyrmex lundi), cultivado em diferentes meios de culturas. Laboratório de Myrmecologia, FAEM/UFPel. Pelotas, RS, 2004.

\begin{tabular}{|c|c|c|c|c|c|c|c|}
\hline Tratamentos & 7 dias & 14 dias & 21 dias & 28 dias & 35 dias & 42 dias & 49 dias \\
\hline $\mathrm{AN}+\mathrm{A}^{*}$ & $0,68 \mathrm{~g}$ & $1,08 \mathrm{~b}$ & 1,60 bc & $2,33 \mathrm{~b}$ & $2,85 \mathrm{~b}$ & $3,32 \mathrm{~b}$ & $3,75 \mathrm{ab}$ \\
\hline $\mathrm{AN}+\mathrm{F}$ & $0,73 \mathrm{f}$ & $1,10 \mathrm{~b}$ & $1,75 \mathrm{~b}$ & $2,33 \mathrm{~b}$ & $2,80 \mathrm{~b}$ & $3,23 \mathrm{~b}$ & 3,48 bc \\
\hline $\mathrm{AN}+\mathrm{T}$ & 0,93 a & $1,50 \mathrm{a}$ & $2,33 \mathrm{a}$ & 2,93 a & 3,53 a & 3,93 a & $4,18 \mathrm{a}$ \\
\hline $\mathrm{BDA}+\mathrm{A}$ & $0,73 \mathrm{e}$ & $1,08 \mathrm{~b}$ & $1,52 \mathrm{~cd}$ & $1,85 \mathrm{~d}$ & $2,15 \mathrm{~d}$ & $2,45 \mathrm{~d}$ & $2,68 \mathrm{e}$ \\
\hline $\mathrm{BDA}+\mathrm{F}$ & 0,75 c & $1,03 \mathrm{~b}$ & $1,38 \mathrm{~d}$ & $1,73 \mathrm{~d}$ & $2,15 \mathrm{~d}$ & $2,63 \mathrm{~cd}$ & 3,00 cde \\
\hline $\mathrm{BDA}+\mathrm{T}$ & $0,78 \mathrm{~b}$ & $1,08 \mathrm{~b}$ & $1,48 \mathrm{~cd}$ & $1,86 \mathrm{~d}$ & $2,20 \mathrm{~d}$ & $2,58 \mathrm{~cd}$ & 2,78 de \\
\hline Pagnocca (Padrão) & $0,74 \mathrm{~d}$ & $1,04 \mathrm{~b}$ & $1,60 \mathrm{bc}$ & $2,08 \mathrm{c}$ & $2,50 \mathrm{c}$ & 2,96 bc & $3,20 \mathrm{~cd}$ \\
\hline CV (\%) & 15,83 & 11,45 & 10,61 & 7,26 & 7,21 & 7,97 & 9,40 \\
\hline
\end{tabular}

Médias seguidas pela mesma letra nas colunas não diferem entre si pelo teste de Tukey a 5\% de probabilidade de erro. *AN + A = Ágar nutritivo + Azevém; AN + F = Ágar nutritivo + Formiga; AN + T = Ágar nutritivo + Tifa; BDA + A = BDA + Azevém; BDA + F = BDA + Formiga; BDA + T = BDA + Tifa.

Ciência Rural, v.36, n.3, mai-jun, 2006. 
Tabela 5 - Peso seco do micélio (mg), aos 53 dias, do fungo Leucoagaricus gongylophorus cultivado pelas formigas Acromyrmex ambiguus, Acromyrmex crassispinus, Acromyrmex heyeri e Acromyrmex lundi, cultivado em diferentes meios de culturas. Laboratório de Myrmecologia, FAEM/UFPel. Pelotas, RS, 2004.

\begin{tabular}{llllll}
\hline Tratamentos & A. ambiguus & A. crassispinus & A. heyeri & A. lundi & CV (\%) \\
\hline AN + A* & $25,45^{* *}$ aA & $22,90 \mathrm{abA}$ & $13,03 \mathrm{aA}$ & $21,38 \mathrm{abA}$ & 22,94 \\
AN + F & $17,06 \mathrm{abA}$ & $15,77 \mathrm{bA}$ & $17,40 \mathrm{aA}$ & $11,08 \mathrm{bcA}$ & 18,17 \\
AN + T & $15,63 \mathrm{abA}$ & $17,48 \mathrm{bA}$ & $11,42 \mathrm{aA}$ & $13,25 \mathrm{abcA}$ & 18,53 \\
BDA + A & $13,15 \mathrm{bA}$ & $14,82 \mathrm{bA}$ & $14,88 \mathrm{aA}$ & $16,18 \mathrm{abcA}$ & 18,19 \\
BDA + F & $15,00 \mathrm{abA}$ & $20,13 \mathrm{abA}$ & $20,68 \mathrm{aA}$ & $14,48 \mathrm{abcA}$ & 18,51 \\
BDA + T & $20,03 \mathrm{abA}$ & $13,42 \mathrm{bAB}$ & $20,62 \mathrm{aA}$ & $7,36 \mathrm{cB}$ & 17,87 \\
Pagnocca (Padrão) & $26,57 \mathrm{aA}$ & $37,85 \mathrm{aA}$ & $11,25 \mathrm{aB}$ & $28,60 \mathrm{aA}$ & 18,98 \\
CV (\%) & 15,25 & 17,22 & 23,28 & 22,17 & \\
\hline
\end{tabular}

Médias seguidas pela mesma letra minúscula nas colunas e maiúscula nas linhas não diferem entre si pelo teste de Tukey a $5 \%$ de probabilidade de erro. * AN + A = Ágar nutritivo + Azevém; AN + F = Ágar nutritivo + Formiga; AN + T = Ágar nutritivo + Tifa; BDA + A $=\mathrm{BDA}+$ Azevém; BDA + F = BDA + Formiga; BDA + T $=$ BDA + Tifa. $* *$ Dados transformados em $\sqrt{(\mathrm{x}+0,5)}$.

Em relação à interação meios de cultura $x$ fungo x períodos de avaliação de diâmetro, o fator meio de cultura foi o mais relevante. A tabela 6 mostra as equações de regressão em função do tempo de avaliação, demonstrando que tanto para as respostas lineares como para as quadráticas, o crescimento fúngico, independente do meio que foi utilizado e de sua procedência, aumenta conforme o passar do tempo. Além disso, comparando-se os valores dos coeficientes de determinação $\left(\mathrm{R}^{2}\right)$, pode-se constatar que os valores são muito próximos e,quase na sua totalidade, acima de $70 \%$.

Tabela 6 - Equações de Regressão, coeficientes de determinação $\left(\mathrm{R}^{2}\right)$ e níveis de probabilidade de erro (P) das interações: meios de cultura X fungos provenientes de diferentes espécies de formigas x tempo de avaliação do diâmetro da colônia.

\begin{tabular}{|c|c|c|c|c|}
\hline Meios & Fungos & Equação & $\mathrm{R}^{2}$ & $\mathrm{P}$ \\
\hline $\mathrm{AN}+\mathrm{A}$ & A. ambiguus & $0,069^{0}+0,092^{5} X-0,0005 X^{2}$ & 97,26 & $* *$ \\
\hline $\mathrm{AN}+\mathrm{A}$ & A. crassispinus & $0,1107+0,0651 X$ & 87,24 & $* *$ \\
\hline $\mathrm{AN}+\mathrm{A}$ & A. heyeri & $0,178^{6}+0,089^{2} X-0,0006 X^{2}$ & 91,81 & $* *$ \\
\hline $\mathrm{AN}+\mathrm{A}$ & A. lundi & $0,1000+0,0761 X$ & 96,30 & $* *$ \\
\hline $\mathrm{AN}+\mathrm{F}$ & A. ambiguus & $0,0971+0,0679 X$ & 96,57 & $* *$ \\
\hline $\mathrm{AN}+\mathrm{F}$ & A. crassispinus & $0,112+0,071 X-0,0003 X^{2}$ & 75,78 & $*$ \\
\hline $\mathrm{AN}+\mathrm{F}$ & A. heyeri & $-0,088^{6}+0,091^{8} X-0,0004 X^{2}$ & 96,13 & $*$ \\
\hline $\mathrm{AN}+\mathrm{F}$ & A. lundi & $-0,1429+0,0957 X-0,0005 X^{2}$ & 97,02 & $*$ \\
\hline $\mathrm{AN}+\mathrm{T}$ & A. ambiguus & $-0,1381+0,1289 X-0,0008 X^{2}$ & 97,72 & $* *$ \\
\hline $\mathrm{AN}+\mathrm{T}$ & A. crassispinus & $0,2543+0,0752 X$ & 97,08 & $* *$ \\
\hline $\mathrm{AN}+\mathrm{T}$ & A. heyeri & $0,2929+0,0852 X$ & 94,08 & $* *$ \\
\hline $\mathrm{AN}+\mathrm{T}$ & A. lundi & $-0,0357+0,1316 X-0,0009 X^{2}$ & 96,66 & $* *$ \\
\hline $\mathrm{BDA}+\mathrm{A}$ & A. ambiguus & $0,2743+0,0761 X-0,0005 X^{2}$ & 96,29 & $*$ \\
\hline $\mathrm{BDA}+\mathrm{A}$ & A. crassispinus & $0,0943+0,0532 X$ & 80,82 & $* *$ \\
\hline $\mathrm{BDA}+\mathrm{A}$ & A. heyeri & $0,5514+0,0497 X$ & 95,03 & $* *$ \\
\hline $\mathrm{BDA}+\mathrm{A}$ & A. lundi & $0,4643+0,0470 X$ & 93,23 & $* *$ \\
\hline $\mathrm{BDA}+\mathrm{F}$ & A. ambiguus & $0,1143+0,0624 X$ & 83,82 & $* *$ \\
\hline $\mathrm{BDA}+\mathrm{F}$ & A. crassispinus & $0,0857+0,0634 X$ & 88,72 & $* *$ \\
\hline $\mathrm{BDA}+\mathrm{F}$ & A. heyeri & $0,2971+0,0670 X$ & 96,69 & $* *$ \\
\hline $\mathrm{BDA}+\mathrm{F}$ & A. lundi & $0,4571+0,0374 X+0,0003 X^{2}$ & 98,51 & $* *$ \\
\hline $\mathrm{BDA}+\mathrm{T}$ & A. ambiguus & $-0,0952+0,1139 X-0,0008 X^{2}$ & 96,72 & $* *$ \\
\hline $\mathrm{BDA}+\mathrm{T}$ & A. crassispinus & $0,3119+0,0557 X$ & 60,82 & $* *$ \\
\hline $\mathrm{BDA}+\mathrm{T}$ & A. heyeri & $-0,1261+0,1288 X-0,0009 X^{2}$ & 98,46 & $* *$ \\
\hline $\mathrm{BDA}+\mathrm{T}$ & A. lundi & $0,4343+0,0496 X$ & 97,40 & $* *$ \\
\hline Pagnocca & A. ambiguus & $0,3464+0,0686 X$ & 96,43 & $* *$ \\
\hline Pagnocca & A. crassispinus & $0,2143+0,0677 X$ & 96,66 & $* *$ \\
\hline Pagnocca & A. heyeri & $0,4881+0,0298 X$ & 82,28 & $* *$ \\
\hline Pagnocca & A. lundi & $0,2857+0,0618 X$ & 91,75 & $* *$ \\
\hline
\end{tabular}

* significativo a $5 \%$ de probabilidade de erro. ${ }^{* *}$ significativo a $1 \%$ de probabilidade de erro.

Ciência Rural, v.36, n3, mai-jun, 2006. 
O fungo simbionte de Atta sexdens L. cresce eficientemente em componentes encontrados nas folhas, como amido e xilana, sendo proposto então, que esses polissacarídeos, quando metabolizados pelo fungo, são importantes fontes de carbono para a dieta das formigas (SIQUEIRA et al., 1998). Isso concorda com os resultados obtidos nos tratamentos ágar nutritivo + tifa, realizados com o fungo cultivado por A. ambiguus (Tabela 1), A. heyeri (Tabela 3) e A. lundi (Tabela 4), nos quais foram observados um diâmetro micelial superior quando comparados a outros tratamentos, no último período de avaliação.

O estudo da simbiose existente entre as formigas cortadeiras e o seu fungo é muito complexo. No entanto, assim como as diferentes espécies de formigas coletam folhas de diferentes espécies de plantas, é provável que o fungo coevoluiu com as mesmas, apresentando-se de forma diferenciada, fazendo com que os mesmos também respondam de maneira diferenciada em diferentes meios de cultura. Por isso, estudos adicionais devem ser realizados para encontrar um meio de cultura que proporcione melhor crescimento deste fungo, o qual, até o momento, apresenta um desenvolvimento lento quando cultivado em laboratório, para futuramente favorecer as pesquisas em torno de seu comportamento.

\section{CONCLUSÃO}

O meio de cultura desenvolvido por PAGNOCCA el al. (1990) mostrou-se o melhor para o desenvolvimento do fungo L. gongylophorus cultivado pelas formigas A. ambiguus, A. crassispinus, A. heyeri e A. lundi.

\section{REFERÊNCIAS}

CAMARGO, R.S. et al. The effect of plant diversity on fungus garden development and foraging behavior of leaf-cutting ants (Hym.: For.). Sociobiology, 42p. p.1-10, 2003.

CHERRETT, J.M. et al. The mutualism between leaf-cutting ants and their fungus. In: WILDING, N.M. et al. (Eds). Insectfungus interactions. London: Academic, 1989. p.93-120.
CURRIE, C.R.; STUART, A.E. Weeding and grooming of pathogens in agriculture by ants. Proceedings of the Royal Society of London B. Biological Sciences, v.263, p.339344, 2001.

DELLA LUCIA, T.M.C. As formigas cortadeiras. Viçosa: Folha de Viçosa, 1993. 262p.

JURUENA, L.F.; CACHAPUZ, L.M.M. Espécies de formigas cortadeiras ocorrentes no Estado do RS. IPAGRO Informa, Porto Alegre, v.23, p.18-24, 1980.

KHOURI, C.R. et al. Efeito de extratos de gramíneas forrageiras no crescimento do fungo simbionte de Acromyrmex (Moellerius) balzani (Hymenoptera, Formicidae). In: SIMPÓSIO DE MIRMECOLOGIA, 16., 2003, Florianópolis. Anais... Florianópolis-SC, 2003. p.483-485.

KOCH, A.L. The kinects of mycelial growth. Journal of General Microbiology, v.89, p.209-216, 1975.

LOECK, A.E. et al. Growth of symbiont fungi of some higher attine ants in mineral medium. Ciência Rural, v.34, p.7982, 2004.

MARTIN, M.M. Invertebrate-microbial interactions. Ithaca: Cornel University, 1987. 148p.

NASCIMENTO, J.S. do. Etiologia, controle e demanda de energia na prevenção da falsa trufa (Diehliomyces microsporus) em cultivos de Agaricus blazei. 2003. $115 \mathrm{f}$. Tese (Doutorado em Energia na Agricultura) - Pós-graduação em Agronomia, Faculdade de Ciências Agronômicas, UNESP.

PAGNOCCA, F.C. et al. Toxicity of sesame extracts to the symbiotic fungus of leaf cutting ants. Bulletin of Entomological Research, v.80, p.349-352, 1990.

SAS Institute. SAS/STAT User's guide, Version 8. Cary, 2000. 1383p.

SIQUEIRA, C.G. de. et al. Metabolism of plant polysaccharides by Leucoagaricus gongylophorus, the symbiotic fungus of the leaf-cutting ant Atta sexdens L. Applied and environmental microbiology, 1998, 64, p. 4820-4822.

VICTOR, S.R. et al. Toxicity of synthetic piperonyl compounds to leaf-cutting ants and their symbiotic fungus. Pest Management Science, v.57, p.603-608, 2001. 